\title{
A prognostic model for stratifying clinical outcomes in chemotherapy-naive metastatic castration-resistant prostate cancer patients treated with abiraterone acetate
}

D. J. Khalaf ${ }^{1}$; C. M. Avilés ${ }^{1}$; A. A. Azad ${ }^{2}$; K. Sunderland ${ }^{1}$; T. Todenhöfer ${ }^{3}$; B. J. Eigl ${ }^{1}$; D. Finch $^{4}$; L. Le ${ }^{5}$; A. Atwell ${ }^{6}$; B. Keith ${ }^{7}$; C. Kollmannsberger ${ }^{1}$; K. N. Chi ${ }^{1,3}$

${ }^{1}$ Department of Medical Oncology, BC Cancer Agency, Vancouver, BC, Canada; ${ }^{2}$ Department of Medicine, Monash University, Clayton, Australia; ${ }^{3}$ Vancouver Prostate Centre, Vancouver, BC, Canada; ${ }^{4}$ Department of Medical Oncology, BC Cancer Agency - Centre for the Southern Interior, Kelowna, BC, Canada; ${ }^{5}$ Department of Medical Oncology, BC Cancer Agency - Fraser Valley Centre, Surrey, BC, Canada; ${ }^{6}$ Department of Medical Oncology, BC Cancer Agency - Vancouver Island Centre, Victoria, BC, Canada; ${ }^{7}$ Department of Medical Oncology, BC Cancer Agency - Abbotsford Centre, Abbotsford, BC, Canada

Cite as: Can Urol Assoc J 2017 Dec. 1; Epub ahead of print. http://dx.doi.org/10.5489/cuaj.4600

Published online December 1, 2017

$* * *$

\section{Abstract}

Introduction: Recently, a prognostic index including six risk factors (RFs) (unfavourable ECOG performance status [PS], presence of liver metastases, short response to luteinizing hormonereleasing hormone [LHRH] agonists/antagonists, low albumin, increased alkaline phosphatase [ALP] and lactate dehydrogenase [LDH]) was developed from the COU-AA-301 trial in postchemotherapy metastatic castration-resistant prostate cancer (mCRPC) patients treated with abiraterone acetate. Our primary objective was to evaluate this model in a cohort of chemotherapy-naive mCRPC patients receiving abiraterone.

Methods: We identified 197 chemotherapy-naive patients who received abiraterone at six BC Cancer Agency centres and who had complete information on all six RFs. Study endpoints were prostate-specific antigen (PSA) response rate (RR), time to PSA progression, time on treatment, and overall survival (OS). PSA RR and survival outcomes were compared using $\mathrm{X}^{2}$ test and logrank test. Multivariable Cox proportional hazard analysis was performed to identify RFs independently associated with OS.

Results: Patients were classified into good (0-1 RFs), intermediate (2-3 RFs), and poor (4-6 RFs) prognostic groups (33\%, 52\%, and 15\%, respectively). For good, intermediate, and poor risk patients, PSA RR ( $\geq 50 \%$ decline) was $60 \%$ vs. $42 \%$ vs. $40 \%$ ( $p=0.05)$; median time to PSA progression was 7.3 vs. 5.3 vs. 5.0 months ( $p=0.02$ ); and median OS was 29.4 vs. 13.8 vs. 8.7 months $(\mathrm{p}<0.0001)$. 
Conclusions: The six-factor prognostic index model stratifies clinical outcomes in chemotherapy-naive mCRPC patients treated with abiraterone. Identifying patients at risk of poor outcomes is important for informing clinical practice and clinical trial design.

\section{Introduction}

The therapeutic landscape for metastatic castration-resistant prostate cancer (mCRPC) has rapidly evolved in recent years with many new agents demonstrating a benefit in overall survival (OS). ${ }^{1}$ One of these agents is abiraterone acetate (abiraterone), an orally available inhibitor of CYP17 that blocks adrenal and intra-tumoural androgen synthesis. Abiraterone confers an OS advantage both as first-line therapy in chemotherapy-naïve patients as well as in the postdocetaxel chemotherapy setting. ${ }^{2,3}$ However, despite its efficacy, outcomes with abiraterone are variable and not all patients derive benefit from treatment. Thus, there is an urgent need for a practical clinical tool able to stratify patients accurately into distinct prognostic categories. In a recent post-hoc analysis of the COU-AA-301 study, a prognostic model was developed for predicting OS in post-docetaxel patients treated with abiraterone. ${ }^{4}$ This model comprises six risk factors (RFs) linked to poor survival: ECOG PS $\geq 2$, liver metastases, time from initiation of androgen deprivation therapy (ADT) to initiation of abiraterone $\leq 36$ months, low albumin $(\leq$ 4g/dl), LDH above upper limit normal (ULN) and alkaline phosphatase (ALP) above ULN. Classification of the COU-AA-301 trial population into good (0-1 RFs), intermediate (2-3 RFs) and poor (4-6 RFs) risk groups revealed OS of 21.3 months, 13.9 months (Hazard Ratio (HR) 2.3) and 6.1 months (HR 6.2) respectively. This model was subsequently validated in a population-based cohort of post-chemotherapy mCRPC patients treated with abiraterone. ${ }^{5}$ The utility of this model in chemotherapy-naïve patients has also been examined in a preliminary analysis incorporating only 64 patients. ${ }^{6}$

The COU-302 trial which evaluated abiraterone in chemotherapy-naïve patients with CRPC showed a significantly longer OS than the post-chemotherapy COU-301 trial (34.7 vs 15.8 months), ${ }^{3,7}$ and the performance of our 6-factor prognostic model in that setting is currently unknown. The aim of our study was to determine whether this prognostic model determines clinically relevant prognostic groups in chemotherapy-naïve patients treated with abiraterone in a real-world setting across six cancer centres in British Columbia, Canada.

\section{Methods}

\section{Patient population}

The BC Cancer Agency (BCCA) consists of six distinct centres and coordinates cancer care delivered throughout British Columbia, Canada. The Cancer Registry at BCCA was reviewed for chemotherapy-naïve mCRPC patients who started abiraterone between July 2009 and October 2014. Patient demographics, prior treatments, clinico-pathological characteristics and outcomes on abiraterone were documented from medical records of each patient. Only patients with available data for all 6 RFs in the prognostic index were included in this study. Research ethics board approval was obtained prior to commencing this study. 


\section{Outcome measures}

Patients were classified into good (0-1 RFs), intermediate (2-3 RFs) and poor (4-6 RFs) risk groups. The following endpoints were determined for each patient: confirmed PSA response rate (PSA decline $\geq 50 \%$ from baseline maintained for $\geq 3$ weeks), time to PSA progression (Prostate Cancer Working Group 2 (PCWG2) criteria), ${ }^{8}$ time on treatment (time from initiation of abiraterone to discontinuation for any reason) and overall survival (OS) (time from initiation of abiraterone to death of any cause or censoring on November $\left.1^{\text {st }}, 2016\right)$. Reasons for discontinuation of abiraterone were recorded as follows: Radiographic (Prostate Cancer Working Group 2 (PCWG2) criteria), biochemical (PCWG2 criteria) or clinical (worsening diseaserelated symptoms requiring a change in anti-neoplastic therapy or a decrease in ECOG PS of $\geq 2$ levels). ${ }^{9,10}$

\section{Development of the prognostic model}

Construction of the six-factor prognostic model has been previously described. ${ }^{4,11}$ In brief, the following steps were involved: i) key clinico-pathological factors were identified and dichotomised for high/low values as necessary; ii) association between baseline clinicopathological factors and OS was investigated using a univariate Cox proportional hazards model; iii) factors that were significant on univariate analysis were incorporated into a multivariate Cox proportional hazards regression model (step-wise procedure); iv) factors that were significant on multivariate analysis were incorporated into the final model, which was then subjected to validation by a bootstrapping approach; v) the C-index was used to determine accuracy of the model, which comprised six separate RFs; and vi) patients were then classified into risk groups based on the number of baseline RFs with median OS calculated for each risk group.

\section{Statistics}

Univariate analysis examining association between prognostic group and PSA response was performed using $\mathrm{X}^{2}$ test. Survival outcomes were estimated using the Kaplan-Meier method. Log-rank test was performed to assess survival differences between groups. Multivariable analysis using Cox proportional hazards model was performed to identify RFs independently associated with OS. Statistical analysis was performed using SPSS ${ }^{\circledR}$ v.14.0 software. To determine the model's accuracy, the c-index was calculated for time on treatment, time to PSA progression and OS.

\section{Results}

\section{Patient population}

Two hundred and forty-six chemotherapy-naïve patients who received abiraterone from July 2009 (when abiraterone became available) until October 2014 were identified. One hundred and ninety-seven patients were included for this analysis as they had available data for each of the 6 RFs comprising the six-factor prognostic model. Patient characteristics at initiation of abiraterone are listed in Table 1. The median age at start of abiraterone was 80 years 
(interquartile range 71-84), 38\% of patients had ECOG PS of 2 or higher and 3\% had liver metastasis. Overall, 33\% (65/197), 52\% (102/197) and 15\% (30/197) of patients were classified as good, intermediate and poor prognosis respectively as per the prognostic index.

Reasons for abiraterone discontinuation (more than one could apply) were clinical progression (36\%), PSA progression (67\%), radiologic progression (33\%) and toxicity (8\%), while 5.5\% $(11 / 197)$ of patients were still on abiraterone as of November $1^{\text {st }}, 2016$. Post-abiraterone systemic treatment was administered to $54 \%$ of patients (100/186).

\section{PSA response}

Confirmed PSA declines $\geq 90 \%, \geq 50 \%$ and $\geq 30 \%$ were seen in $13 \%(26 / 197), 48 \%(94 / 197)$ and 51\% (100/197) of patients respectively. All three levels of PSA decline were significantly more frequent in good versus intermediate and poor-risk patients (Table 2). Only ECOG PS was significantly associated with PSA decline $\geq 50 \%$ on univariate analysis ( $\mathrm{X}^{2}$ test).

\section{Survival endpoints}

For the overall cohort, median time to PSA progression, median time on treatment and median OS was 6.5 months (95\% CI 5.6-8.0), 7.4 months (95\% CI 6.0-8.5) and 15.7 months (95\% CI 12.9-18.9) respectively. As shown in Table 2, time to PSA progression and time on treatment were significantly longer in the good prognosis group versus the intermediate and poor risk groups ( $P=0.02$ and $P<0.0001$ respectively; log-rank). Hazard ratios for each group are shown in Table 3. OS was significantly better in the good prognosis group compared with the intermediate and poor prognostics groups (29.4 vs 13.8 vs 8.7 months, respectively, $P<0.0001)$. Kaplan-Meier curves are shown in Figure 1. On multivariable analysis incorporating the 6 RFs from the prognostic index, ECOG PS $(P<0.0001)$, liver metastases $(P=0.0008)$, time from initiation of ADT to abiraterone $\leq 36$ months $(P=0.02)$ and serum ALP $(P<0.0001)$ were confirmed as independent prognostic factors for OS, whereas serum albumin and serum LDH did not meet statistical significance (Table 4). The predictive accuracy of our model as measured by the c-index was $0.79,0.68$ and 0.66 for OS, time on treatment and time to PSA progression, respectively.

\section{Discussion}

Although abiraterone has proven efficacy in chemotherapy-naïve patients with mCRPC, treatment outcomes are variable and difficult to predict at an individual patient level. Recently, a prognostic model for OS in patients receiving abiraterone after prior docetaxel chemotherapy was developed based on data from the COU-AA-301 phase III trial, and subsequently validated in a population based cohort. ${ }^{4,5}$ In the present study, we confirm the performance and discriminatory power of this model in a large, unselected and sequential cohort of chemotherapynaïve mCRPC patients treated with abiraterone. Our real-world population differed significantly from patients in the COU-AA-302 study which established the efficacy of abiraterone in chemotherapy-naïve patients. We included patients with poor performance status, significant pain symptoms and visceral metastasis, all of which were exclusion criteria for the COU-AA-302 
study. This is reflected in the difference between the median overall survival of our cohort (15.7 months) compared to the median overall survival observed in the COU-AA-302 study (34.7 months). ${ }^{3}$ In addition, a lower proportion of our patients had low risk versus intermediate or high risk prognostic scores ( $33 \%$ vs $67 \%$ respectively), compared to the COU-AA-301 study ( $46 \%$ vs $53 \%){ }^{4}$ This also further emphasizes the applicability and generalizability of the prognostic model to the broader patient population treated with abiraterone.

We observed a clear and statistically significant difference in OS between good, intermediate and poor-risk patients and confirmed that presence of liver metastases, time from initiation of ADT to initiation of abiraterone $\leq 36$ months, serum ALP $\geq$ ULN and ECOG PS $\geq 2$ are independent prognostic factors. Interestingly, serum LDH and serum albumin showed no independent prognostic value for OS, although this is likely due to the limited sample size of our study. These results contrast with those of an external validation of the prognostic index performed by Ravi et al at Royal Marsden. ${ }^{6}$ In their cohort of 64 chemotherapy-naïve patients, low albumin was the only independent factor predicting OS in multivariable analysis. However, this was a relatively small validation cohort which included only one chemotherapy-naïve patient with poor prognosis disease, and differed from our cohort in that no chemotherapy-naïve patients with ECOG PS $\geq 2$ were included whereas these patients comprised $38 \%$ of our cohort. The different cohort characteristics and larger sample size may also account for the better predictive power of the model in our study as Ravi et al observed a relatively modest OS difference of 10 months between good and intermediate/poor-risk patients.

There is strong evidence from other studies supporting the prognostic value of the clinical factors included in our model. A prognostic nomogram was developed and validated by Halabi et al from two randomized controlled trials of first-line docetaxel for mCRPC. ${ }^{12}$ Their analysis also showed that ECOG PS, site of metastasis, LDH, albumin, hemoglobin and ALP predict OS on multivariable analysis, in addition to opioid analgesia use and PSA. However, they did not test the prognostic significance of duration of primary ADT when building their model, and its performance in the setting of first-line abiraterone has not been verified. In a study of 161 patients treated with abiraterone, McKay et al demonstrated that duration of primary ADT $>12$ months and no prior docetaxel chemotherapy were independently associated with a longer time on abiraterone. ${ }^{13}$ In addition, a recent meta-analysis evaluating the impact of site of metastasis on overall survival in men with CRPC showed that liver metastasis predicted worse OS compared to bone metastasis and lymph node only metastasis (13.5 vs 21.3 vs 31.6 months, respectively). ${ }^{14}$ In addition to OS, we observed that the 6-factor prognostic index model is predictive of time to PSA progression, PSA response and time on treatment in chemotherapy-naïve patients treated with abiraterone. The use of PSA parameters as a surrogate endpoint for OS in CRPC has generated considerable discussion and controversy. However recently, Xu et al constructed a biomarker-survival modelling framework to explore the relationship between PSA kinetics (including time to PSA progression and PSA response) and OS in metastatic CRPC patients following administration of abiraterone. ${ }^{15}$ In their analysis, which was based on data from the COU-AA-301 and COU-AA-302 trials, PSA kinetics were highly associated with OS in both 
chemotherapy-experienced patients and chemotherapy-naïve patients. The authors concluded that PSA kinetics should be considered as surrogate end points of clinical benefit in abirateronetreated patients regardless of chemotherapy treatment. ${ }^{15}$ Our observation that the prognostic model predicted for both PSA response and OS in the present cohort is in accordance with this data. Our model was also predictive for time on treatment, a useful surrogate in the real-world setting for the duration of clinical benefit on treatment and an important endpoint, as highlighted in the updated recommendations on trial design and objectives from the Prostate Cancer Working Group 3. ${ }^{16}$ These findings demonstrate that our model is predictive of outcomes on abiraterone and thus may be valuable in selecting patients likely to benefit from first-line abiraterone, and identifying those for whom alternate treatments could be considered, such as first-line chemotherapy or clinical trials.

A key strength of the 6-factor prognostic index model is that it is a pragmatic tool for risk stratification utilising easily available (and inexpensive) clinical parameters. Nevertheless, integration of this model with emerging molecular biomarkers including androgen receptor (AR) splice variants, circulating tumour DNA (ctDNA) and circulating tumour cells (CTCs) will be important. AR splice variant 7 (ARv7) detection in CTCs was recently proposed as a mechanism driving primary resistance to enzalutamide and high levels of full-length AR mRNA and presence of ARv7 have been shown to correlate with PSA progression-free survival and OS on abiraterone or enzalutamide. ${ }^{17,18}$ Recent evidence has revealed that structural variants of the AR gene are associated with the presence of AR splice variants, and may also be important drivers of treatment resistance. ${ }^{19}$ In addition, AR gene aberrations (copy number change and/or mutations) in pre-treatment ctDNA have been linked to adverse outcomes in mCRPC patients commencing abiraterone and enzalutamide. ${ }^{20,21}$ A biomarker panel incorporating CTC enumeration and LDH was also recently shown to predict OS in a post-hoc analysis of the COU-AA-301 trial. ${ }^{22}$ The present study has various limitations. These include its retrospective design, being limited to a single province in Canada and the relatively small sample size. We could not assess radiological response to treatment since imaging was not consistently performed. Data on radiographic progression-free survival were not analysed due to wide variation in follow-up including timing of imaging.

\section{Conclusion}

In conclusion, we observe that the 6-factor prognostic index model provides reliable risk stratification for chemotherapy-naïve patients receiving abiraterone by predicting PSA response, time to PSA progression, time on treatment and OS. ECOG PS, liver metastases, time from androgen deprivation therapy (ADT) to initiation of abiraterone $\leq 36$ months and serum ALP were confirmed as independent risk factors for poor OS in the pre-chemotherapy setting. Due to its predictive capability, we suggest that incorporating the prognostic model in clinical practice and future trials assessing the use of novel hormonal agents and cytotoxics will allow improved risk stratification and optimized treatment selection. 


\section{References}

1. Heidenreich A, Pfister D, Merseburger A, et al; for German Working Group on Castration-Resistant Prostate Cancer (GWG-CRPC). Castration-resistant prostate cancer: Where we stand in 2013 and what urologists should know. Eur Urol 2013;64:260-5.

2. de Bono JS, Logothetis CJ, Molina A, et al. Abiraterone and increased survival in metastatic prostate cancer. N Engl J Med 2011;364:1995-2005.

3. Ryan CJ, Smith MR, Fizazi K, et al. Abiraterone acetate plus prednisone versus placebo plus prednisone in chemotherapy-naive men with metastatic castration-resistant prostate cancer (COU-AA-302): Final overall survival analysis of a randomised, double-blind, placebo-controlled phase 3 study. Lancet Oncol 2015;16:152-60.

4. Chi KN, Kheoh T, Ryan CJ, et al. A prognostic index model for predicting overall survival in patients with metastatic castration-resistant prostate cancer treated with abiraterone acetate after docetaxel. Ann Oncol 2016;27:454-60.

5. Azad A, Lester R, Leibowitz-Amit R, et al. Population-based analysis of a novel prognostic model for metastatic castration-resistant prostate cancer (mCRPC) patients (pts) treated with abiraterone acetate (AA) [abstract]. J Clin Oncol 2014;32:5S.

6. Ravi P, Mateo J, Lorente D, et al. External validation of a prognostic model predicting overall survival in metastatic castrate-resistant prostate cancer patients treated with abiraterone. Eur Urol 2014;66:8-11.

7. Fizazi K, Scher HI, Molina A, et al. Abiraterone acetate for treatment of metastatic castration-resistant prostate cancer: Final overall survival analysis of the COU-AA-301 randomised, double-blind, placebo-controlled phase 3 study. Lancet Oncol 2012;13:98392.

8. Scher HI, Halabi S, Tannock I, et al. Design and end points of clinical trials for patients with progressive prostate cancer and castrate levels of testosterone: Recommendations of the prostate cancer clinical trials working group. J Clin Oncol 2008;26:1148-59.

9. Azad AA, Eigl BJ, Murray RN, et al. Efficacy of enzalutamide following abiraterone acetate in chemotherapy-naive metastatic castration-resistant prostate cancer patients. Eur Urol 2015;67:23-29.

10. Azad AA, Leibowitz-Amit R, Eigl BJ, et al. A retrospective, canadian multi-center study examining the impact of prior response to abiraterone acetate on efficacy of docetaxel in metastatic castration-resistant prostate cancer. Prostate 2014;74:1544-50.

11. Azad AA, Eigl BJ, Leibowitz-Amit R, et al. Outcomes with abiraterone acetate in metastatic castration-resistant prostate cancer patients who have poor performance status. Eur Urol 2015;67:441-47.

12. Halabi S, Lin CY, Kelly WK, et al. Updated prognostic model for predicting overall survival in first-line chemotherapy for patients with metastatic castration-resistant prostate cancer. J Clin Oncol 2014;32:671-77. 
13. McKay RR, Werner L, Fiorillo M, et al. Predictors of duration of abiraterone acetate in men with castration-resistant prostate cancer. Prostate Cancer Prostatic Dis 2016;19:398-405.

14. Halabi S, Kelly WK, Ma H, et al. Meta-analysis evaluating the impact of site of metastasis on overall survival in men with castration-resistant prostate cancer. J Clin Oncol 2016;34:1652-59.

15. Xu XS, Ryan CJ, Stuyckens K, et al. Correlation between prostate-specific antigen kinetics and overall survival in abiraterone acetate-treated castration-resistant prostate cancer patients. Clin Cancer Res 2015;21:3170-77.

16. Scher HI, Morris MJ, Stadler WM, et al. Trial design and objectives for castrationresistant prostate cancer: Updated recommendations from the prostate cancer clinical trials working group 3. J Clin Oncol 2016;34:1402-18.

17. Antonarakis ES, Lu C, Wang $\mathrm{H}$, et al. AR-V7 and resistance to enzalutamide and abiraterone in prostate cancer. N Engl J Med 2014;371:1028-38.

18. Silberstein J, Luber B, Wang $\mathrm{H}$, et al. Clinical significance of AR mRNA quantification from circulating tumor cells (CTCs) in men with metastatic castration-resistant prostate cancer (mCRPC) treated with abiraterone (abi) or enzalutamide (enza) [abstract]. $J$ Clin Oncol 2017;35:6S.

19. De Laere B, van Dam PJ, Whitington T, et al. Comprehensive profiling of the androgen receptor in liquid biopsies from castration-resistant prostate cancer reveals novel intraAR structural variation and splice variant expression patterns. Eur Urol. In press 2017.

20. Romanel A, Gasi Tandefelt D, Conteduca V, et al. Plasma AR and abiraterone-resistant prostate cancer. Sci Transl Med 2015;7:312re10.

21. Azad AA, Volik SV, Wyatt AW, et al. Androgen receptor gene aberrations in circulating cell-free DNA: Biomarkers of therapeutic resistance in castration-resistant prostate cancer. Clin Cancer Res 2015;21:2315-24.

22. Scher HI, Heller G, Molina A, et al. Circulating tumor cell biomarker panel as an individual-level surrogate for survival in metastatic castration-resistant prostate cancer. $J$ Clin Oncol 2015;33:1348-55. 


\section{Figures and Tables}

Fig. 1. Kaplan-Meier curves for: (A) overall survival; (B) time on treatment ; and (C) PSA progression for good, intermediate, and poor prognosis groups.

A

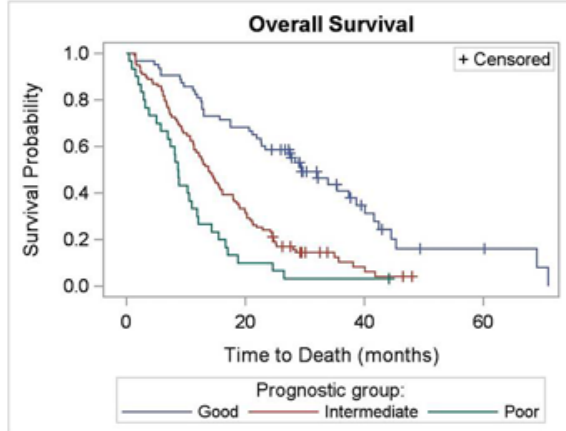

B

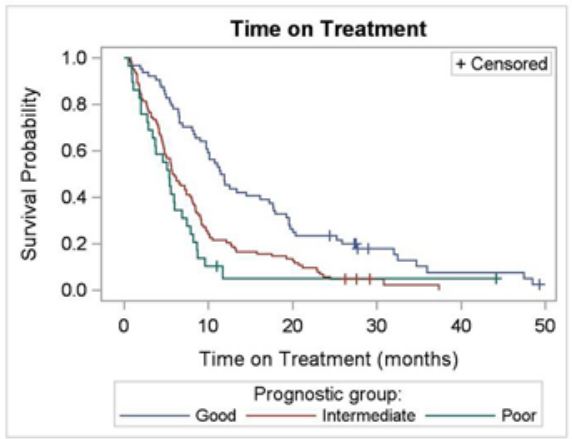

C

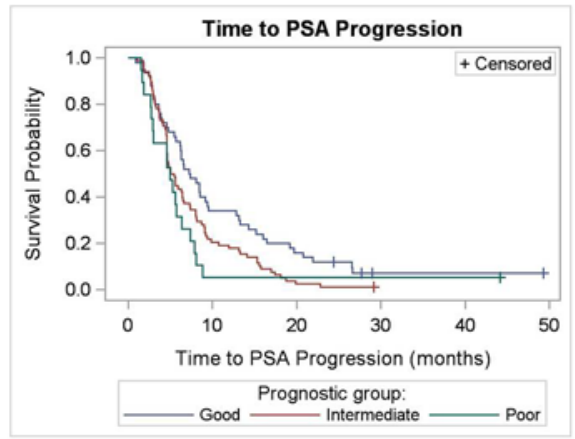




\begin{tabular}{|c|c|}
\hline \multicolumn{2}{|c|}{$\begin{array}{l}\text { Table 1. Baseline characteristics at initiation of } \\
\text { abiraterone acetate }(n=197) \\
\text { Characteristic }\end{array}$} \\
\hline \multicolumn{2}{|l|}{ Age } \\
\hline Median (IQR) & $80(71-84)$ \\
\hline \multicolumn{2}{|l|}{ Gleason score } \\
\hline $4-5$ & $5(3)$ \\
\hline $6-7$ & $49(25)$ \\
\hline $8-10$ & $109(55)$ \\
\hline Unknown & $34(17)$ \\
\hline \multicolumn{2}{|l|}{ Disease sites, n (\%) } \\
\hline Bone & $149(76)$ \\
\hline Lymph node & $58(29)$ \\
\hline Liver & $5(3)$ \\
\hline \multicolumn{2}{|l|}{ Time since commencing ADT } \\
\hline Median (months, IQR) & $47(22-93)$ \\
\hline \multicolumn{2}{|l|}{ ECOG PS, n (\%) } \\
\hline $0-1$ & $122(62)$ \\
\hline$\geq 2$ & $75(38)$ \\
\hline \multicolumn{2}{|l|}{ Bone pain $^{\mathrm{a}}$, n (\%) } \\
\hline Yes & $65(33)$ \\
\hline 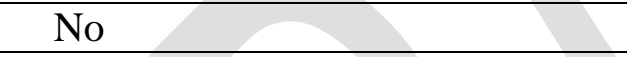 & $129(66)$ \\
\hline \multicolumn{2}{|l|}{ Disease progression, n (\%) } \\
\hline PSA & $175(89)$ \\
\hline Clinical & $105(53)$ \\
\hline Radiographic & $99(50)$ \\
\hline \multicolumn{2}{|l|}{ Laboratory } \\
\hline LDH (median, U/L) (IQR) & $234(179-361)$ \\
\hline Elevated ( $\geq$ ULN), n (\%) & $55(28)$ \\
\hline ALP (median, U/L) (IQR) & $113(80-221)$ \\
\hline Elevated ( $\geq$ ULN), n (\%) & $69(35)$ \\
\hline Albumin (median, U/L) (IQR) & $38(35-41)$ \\
\hline Low (<4g/dl), n (\%) & $130(66)$ \\
\hline Hemoglobin (median, U/L) (IQR) & $123(111-132)$ \\
\hline Low $(<100)$, n $(\%)$ & $22(11)$ \\
\hline
\end{tabular}

${ }^{a}$ Bone pain defined as requiring radiotherapy and/or opioid analgesia within 28 days of commencing abiraterone. ADT: androgen-deprivation therapy (LHRH agonist or antagonist for metastatic or relapse disease); ECOG PS: Eastern Cooperative Group Performance Status; IQR: interquartile range. 


\begin{tabular}{|c|c|c|c|c|}
\hline Parameter & Good prognosis & Intermediate prognosis & Poor prognosis & $\mathbf{p}$ \\
\hline & $(0-1$ RF) $(n=65)$ & $(2-3$ RF) $(n=102)$ & $(4-6$ RF) $(n=30)$ & \\
\hline \multicolumn{5}{|l|}{ PSA decline $^{\mathrm{a}}$} \\
\hline Decline $\geq 90, \mathrm{n}(\%)$ & $14(22)$ & $11(11)$ & $1(3)$ & 0.04 \\
\hline Decline $\geq 50, \mathrm{n}(\%)$ & $39(60)$ & $43(42)$ & $12(40)$ & 0.05 \\
\hline Decline $\geq 30, \mathrm{n}(\%)$ & $40(62)$ & $46(45)$ & $14(47)$ & 0.10 \\
\hline \multicolumn{5}{|l|}{ Time on treatment } \\
\hline Median (month, 95\% CI) & $11.6(9.7-17.2)$ & $5.8(4.8-7.8)$ & $5.3(2.9-6.9)$ & $<0.0001$ \\
\hline \multicolumn{5}{|l|}{ Time to PSA progression ${ }^{b}$} \\
\hline Median (months, 95\% CI) & $7.3(5.7-9.4)$ & $5.3(4.6-6.5)$ & $5.0(2.8-6.4)$ & 0.02 \\
\hline \multicolumn{5}{|l|}{ Overall survival $^{\mathrm{c}}$} \\
\hline Median (months, 95\% CI) & $29.4(22.6-38.7)$ & $13.8(11.4-16.1)$ & 8.7 (5.8-11.9) & $<0.0001$ \\
\hline
\end{tabular}

${ }^{\mathrm{a}}$ PSA decline confirmed $\geq 3$ weeks later; ${ }^{\mathrm{b}} \mathrm{PCWG} 2$ criteria; ${ }^{\mathrm{c}}$ from time of commencing abiraterone. CI: confidence interval; PSA: prostate-specific antigen; RF: risk factors (including: ECOG performance status $\geq 2$; liver metastases; time on androgen-deprivation therapy to initiation of abiraterone $\leq 36$ months; low albumin ( $<4 \mathrm{~g} / \mathrm{dl}$ ); high LDH (>upper limit normal); and high ALP (>upper limit normal).

Table 3. Hazard ratios for time on treatment, time to PSA progression, and overall survival stratified by prognostic group

\begin{tabular}{|c|c|c|c|}
\hline & \multicolumn{3}{|c|}{ Hazard ratio $^{\mathrm{a}}$} \\
\hline Parameter & Good (0-1 RF) & Intermediate (2-3 RF) & $\begin{array}{c}\text { Poor (4-6 } \\
\text { RF) }\end{array}$ \\
\hline Time on treatment $(95 \% \mathrm{CI})$ & - & $2.0(1.4-2.8)$ & $2.7(1.7-4.3)$ \\
\hline Time to PSA progression ${ }^{\mathrm{b}}(95 \% \mathrm{CI})$ & - & $1.5(1.1-2.2)$ & $2.0(1.1-3.4)$ \\
\hline Overall survival $^{\mathrm{C}}(95 \% \mathrm{CI})$ & - & $2.5(1.7-3.6)$ & $4.4(2.7-7.1)$ \\
\hline
\end{tabular}

${ }^{\mathrm{a}}$ Relative to good prognosis group; ${ }^{\mathrm{b}} \mathrm{PCWG} 2$ criteria; ${ }^{\mathrm{C}}$ from time of commencing abiraterone. CI: confidence interval; PSA: prostate-specific antigen; RF: risk factors (including: ECOG performance status $\geq 2$; liver metastases; time on androgen-deprivation therapy to initiation of abiraterone $\leq 36$ months; low albumin (<4g/dl); high LDH (>upper limit normal); and high ALP (>upper limit normal). 


\begin{tabular}{|c|c|c|c|}
\hline Characteristic & HR & 95\% CI & $\mathbf{p}$ \\
\hline Time on ADT & 1.5 & $1.1-2.1$ & 0.02 \\
\hline ECOG PS & 2.2 & $1.6-3.1$ & $<0.0001$ \\
\hline Serum LDH & 1.2 & $0.8-1.7$ & 0.39 \\
\hline Serum ALP & 2.1 & $1.5-3.0$ & $<0.0001$ \\
\hline Liver metastases & 5.0 & $1.9-12.7$ & 0.0008 \\
\hline Serum albumin & 1.0 & $0.7-1.4$ & 0.88 \\
\hline
\end{tabular}

ADT: androgen-deprivation therapy; ALP: alkaline phosphatase; CI: confidence interval; HR: hazard ratio; LDH: lactate dehydrogenase; mCRPC: metastatic castration-resistant prostate cancer. 
